\title{
Death receptor-mediated suicide: a novel target of autoimmune disease treatment
}

\author{
Thomas Brunner, Diana Arnold, Christoph Wasem, Jean A. \\ Laissue \& Christoph Mueller
}

Division of Immunopathology, Institute of Pathology, University of Berne,

\section{Review}

1. Introduction

2. The T-cell as a major target of autoimmune disease treatment

3. Role and mechanisms of activation-induced suicide in immune regulation and prevention of autoimmune diseases

4. Regulation of AICD, CD95- and TNF receptor-induced apoptosis

5. Bisindolylmaleimide VIII: a novel and prototypic drug for the treatment of autoimmune diseases

5. Conclusion and expert opinion

Acknowledgments

Bibliography Murtenstrasse 31, 3010 Berne, Switzerland

In the thymus, based on the reactivity of their T-cell receptor with self-MHC and antigenic peptides, developing immature T-cells undergo positive and negative selection. Cells recognising self-peptides and MHC with high affinity are considered autoreactive, and thus potentially harmful, and are eliminated by induction of apoptotic cell death. Thymic negative selection is, however, only incomplete and autoreactive T-cells escape into the periphery. It is not the presence of autoreactive mature T- and B-lymphocytes as the underlying cause of tissue destruction and development of autoimmune diseases, but their uncontrolled and excessive clonal expansion upon activation by self-antigen. Thus, potent regulatory mechanisms must keep these autoreactive cells under control to avoid their inappropriate activation. Recent evidence indicates that death receptors of the tumour necrosis factor receptor family play a central role in mediating antigen receptor-induced suicide of autoreactive T-lymphocytes. Defects in these apoptosis-inducing regulatory mechanisms may result in the development of autoimmune diseases. Therefore, enhancing the cell's own suicide program, offers a most attractive therapeutic target for the treatment of autoimmune diseases.

Keywords: activation-induced cell death, apoptosis, autoimmune disease, $B c l-2, B c l-x_{\mathrm{L}}$, caspases, CD95, death receptors, Fas, gld, inhibitor of apoptosis (IAP), Fas-associated death domain (FADD), Fas-associated protein-1

(FAP-1), Flice-like inhibitory protein (FLIP), lpr, NFкB, protein kinase C, T-lymphocytes, TNF- $\alpha$

\section{Introduction}

Many typical infectious diseases, such as those initiated and mediated by invading and parasiting viruses, bacteria and other pathogens, can successfully be fought by activating the body's own defence mechanism, the immune system, as by vaccination for example. In autoimmune diseases, the enemy lies within. It is the uncontrolled action of this otherwise mostly beneficial and protective immune system, that causes a variety of different forms of self-destruction. The decision criteria that make the glorious defender of our body a corrupt betrayer have been the topic of extensive research. Whereas we currently understand many aspects of autoimmune disease development, the essential breakthroughs in the treatment of autoimmune diseases are still awaiting, and few successful therapeutic interventions are currently established. Thus, novel and potentially also 
unconventional strategies are urgently needed for clinical application.

This review will focus on a novel target of autoimmune therapy: the T-lymphocyte's own suicide program. Besides its novelty, it represents the logical consequence of our increasing knowledge of how immune responses are normally regulated and terminated: the removal of undesired cells by apoptotic cell death. To estimate the potential role and usefulness of this therapeutic target, common aspects of all autoimmune diseases and the cellular and molecular bases of their destructive forces should first be discussed.

In general, all autoimmune diseases result in the destruction of tissues and cells that are vital for the functioning of the body (reviewed in [1]). A major aspect in this process is the crucial involvement of the immune system and the specific recognition of self-antigens by B- and T-cells. This specific recognition will initiate a rather uncontrolled immune response against self-antigens. Cells expressing these structures may either be direct targets of cell-mediated cytotoxicity or of auto-antibodies. This specific recognition of self-antigen initiating an autoimmune response appears to be a key element in the development of autoimmune diseases, and is reflected by the fact that many autoimmune diseases have a genetic predisposition, i.e. susceptibility to the disease can be correlated with certain HLA phenotypes [2]. Yet, although the presence of autoreactive T-cells and B-cells can be easily demonstrated in most individuals, autoimmune tissue destruction, with subsequent clinical onset of an autoimmune disorder, is a rare event. This suggests that efficient control mechanisms exist to prevent autoreactive lymphocytes from being activated and mounting a destructive immune response against self.

What are these control mechanisms, how do they work, why do they fail sometimes? Can they be targeted for efficient treatment of autoimmune diseases? The answers to these questions may well form the basis for the development of novel therapies.

\section{The T-cell as a major target of autoimmune disease treatment}

Extensive research has been focussing on the role of T-cells in the development of autoimmune disease [3-5]. T-cells specifically recognise antigen with their
T-cell receptors. Their activation leads to the development of effector functions. In the case of autoreactive T-cells, this may result in cell-mediated cytotoxicity and subsequent tissue destruction. In addition, the helper function for B-cells enhances production of auto-antibodies and further tissue destruction. Although B-cells can recognise certain antigens directly via the B-cell receptor, the recognition of many antigens by $\mathrm{B}$-cells requires $\mathrm{T}$-cell help (costimulation and cytokines) for successful antibody production and immunoglobulin class switch. Thus, irrespective of the effector mechanisms involved in the autoimmune destruction process, the T-cell is an important orchestrating cell type of the immune system, and thus represents a major target for the modulation of most immune responses, including those involved in autoimmune diseases. Therefore, a successful therapy should be aimed at regulating, eliminating or suppressing these autoreactive and disease promoting T-cells.

During their development in the thymus, immature lymphocytes will acquire a T-cell receptor with a defined, but random, specificity. Upon further intrathymic differentiation, those immature thymocytes recognising self-antigens in the context of MHC (HLA) molecules with high affinity are eliminated through induction of apoptosis. As evidenced by the frequent detection of potentially autoreactive T-cells in peripheral blood this negative selection of thymocytes is not complete. Some potentially autoreactive T-cells may escape this central deletion and migrate to peripheral lymphoid organs. However, autoreactive T-cells appear not only to escape from thymic negative selection in patients suffering from autoimmune disease, but can also be detected in healthy individuals (reviewed in [5]). Whereas autoreactivity can be demonstrated in vitro, certain tolerance mechanisms appear to control the activation and expansion of these cells in vivo. It is the restoration of this self-tolerance that has been the aim of most therapies of autoimmune diseases. Thus, in experimental systems of autoimmune disease, a variety of T-cell tolerising protocols have been developed and applied for the treatment of the disease, with variable success. For example, oral administration, or repeated iv. injection of immunodominant self-peptides has been used to tolerise autoreactive T-cells and to prevent experimental allergic encephalomyelitis (EAE), a murine model for multiple sclerosis in humans (reviewed in [6]). Similarly, administration of anti-T cell receptor (TCR) 
or anti-CD4 antibodies has been shown to ameliorate the disease progression in animal models of, but also in, patients with rheumatoid arthritis (reviewed in [7]). Activation of these autoreactive T-cells in a tolerising context appears to induce functional unresponsiveness most likely through several distinct mechanisms, including the induction of apoptotic cell death [8]. Altered peptide ligands or competitor peptides have been also used to compete with the presentation of the endogenous disease-inducing self-peptides to induce a stage of unresponsiveness in autoreactive T-cells [9]. In principle, all of these approaches target the specific recognition of the self-antigen by the T-cell and, therefore, the three-molecular complex consisting of TCR, MHC and antigenic peptide. It is the specificity of a given TCR that would theoretically allow individualisation of antigen-specific therapy without generally compromising immune surveillance, as it may happen when using immunosuppressive drugs such as cyclosporin A and FK506 (reviewed in [10]). Although in experimental systems, such as EAE, therapies based on peptidespecific tolerance induction have been proven to be successful [6], the general application of this strategy in different autoimmune diseases is likely to encounter problems. Most of the auto-antigens that cause the different autoimmune diseases remain unidentified. Their identification represents a difficult and laborious task. In addition, genetic differences, e.g. in MHC and therefore peptide binding and TCRs that recognise these ligands, would require an individualisation of a peptide-based tolerance induction protocol. Thus, although induction of unresponsiveness in autoreactive T-cells by tolerisation may be promising for the treatment of a limited number of autoimmune diseases, it may be unsuitable for other diseases for certain individuals. Hence, additional strategies have to be developed.

\section{Role and mechanisms of activation-induced suicide in immune regulation and prevention of autoimmune diseases}

During an immune response, antigen-specific T-cells expand quickly and may comprise a high percentage of all cells present in lymphoid organs, as has been shown during viral infections [11,12]. Once the antigen has been cleared, this large population of reactive T-cells has to be reduced efficiently to avoid tissue damage, possibly leading to the development of autoimmune disease. It is generally believed that induction of apoptotic cell death and subsequent phagocytosis of the dying cells is responsible for the removal of chronically activated T-lymphocytes at the end of an immune response, and thus for the maintenance of immune homeostasis (reviewed in [13-15]). Whereas activation of resting T-cells results in proliferation and development of effector function, TCR activation of primed T-cells induces apoptosis. This process is termed activation-induced cell death (AICD) and deregulation of this suicide program may result in severe damage of tissue and organs. Recently, the molecular events leading to AICD have been uncovered. Restimulation of primed T-cells leads to expression of the apoptosis-inducing receptor CD95 (Fas/APO-1) and its ligand (CD95L) and their subsequent interaction induces apoptosis [16-20]. AICD may act in a paracrine way, as activated CD95L expressing T-cells can kill CD95 expressing neighbouring cells, but also in an autocrine manner. CD95 and its ligand may be expressed by, and act on, the same cell, as has been shown by single cell analysis $[16,18,21]$. Therefore, it has been suggested that membrane blebs containing CD95L may be released from the cells and may bind back to the cytoplasmic membrane bound receptor, activating the apoptosis machinery and resulting in subsequent death $[16,18,22]$. It has been shown that CD95L can be released from the cell surface by metalloproteases [23-26]. However, it is unlikely that soluble CD95L is responsible for this phenomenon of $\mathrm{T}$-cell suicide, since soluble CD95L, rather inefficiently, triggers apoptosis and down-modulates cell surface CD95 levels [24,26]. Therefore, soluble CD95L most probably exhibits a negative regulatory function, similar to soluble CD95 receptor [27-29]. The importance of CD95/CD95L interaction in downsizing the pool of antigen-reactive T-lymphocytes after an immune response in vivo is demonstrated by the fact that the natural mutant mouse strain for CD95 (lpr), shows defective AICD in response to antigen administration in vivo [30-34]. Therefore, antigen-reactive T-cells persist for a prolonged period of time. Whereas AICD in $\mathrm{CD}_{4}{ }^{+} \mathrm{T}$-cells appears to be mostly mediated through CD95/CD95L interaction [16,28-20], activation-induced suicide of $\mathrm{CD}^{+}$T-cells also involves expression of TNF- $\alpha$ and TNF receptors) [35-36] (See Figure 1).

CD95 and TNF receptor I and II belong to the family of TNF-/nerve growth factor receptor-like homologous molecules, all intimately involved in either mediating 
Figure 1: Death receptor-mediated suicide in $\mathrm{CD} 4^{+}$and $\mathrm{CD} 8^{+} \mathrm{T}$-cells: chronic activation of T-cells via the T-cell receptor (TCR) leads to expression of CD95 ligand (CD95L) and TNF- $\alpha$, and their corresponding receptors, CD95 and TNF receptor (TNFR). Subsequent interaction of ligands with their receptors induces apoptotic death. CD95L and TNF- $\alpha$ may act in a cell-autonomous way (autocrine) or may induce apoptosis a paracrine manner. Soluble CD95L binds to membrane CD95, but does not trigger apoptosis. APC:Antigen-presenting cell

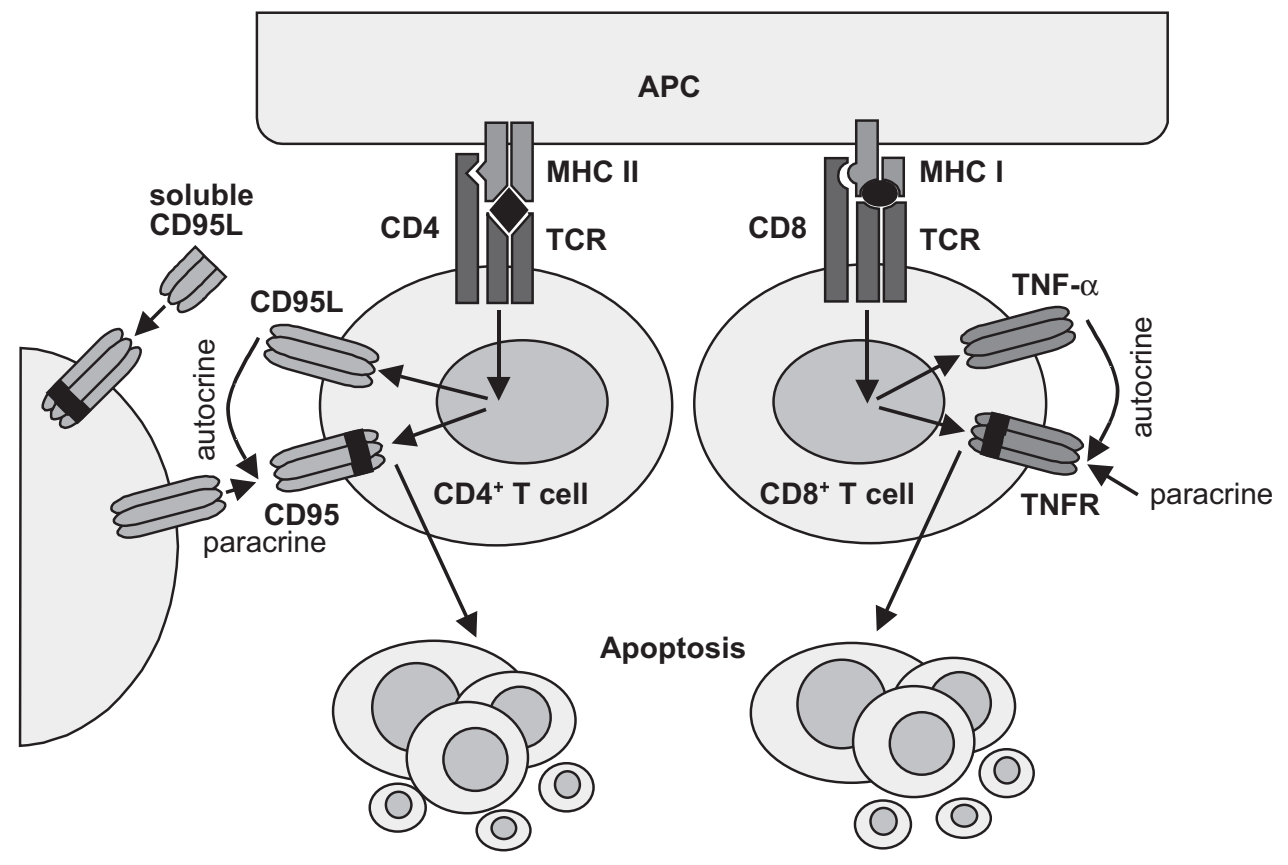

apoptosis or survival. CD95 and TNF receptors appear to be the most relevant apoptosis-inducing receptors in T-cell regulation, whereas other members of this family, also capable of activating the death machinery (such as death receptor (DR) 3, 4 and 5), are probably less important [37].

Autoreactive T-cells that have escaped thymic negative selection may fail to encounter antigen for the duration of their life, due to inaccessibility of the antigen (e.g., proteins with highly tissue specific expression, such as insulin), and may persist in a resting state until antigen may be uncovered (e.g., after tissue damage) [38]. In contrast, some autoreactive T-cells may constantly be exposed to self-antigens presented by professional or non-professional antigen-presenting cells. Whereas autoreactive T-cells may be relatively frequent, the development of autoimmune pathologies is still a remarkably rare event. It must be questioned as to what prevents these autoreactive T-cells from causing autoimmune disorders. There is increasing evidence that the same mechanisms that control the extent of an immune response, also regulate autoreactive T-cells. Autoreactive T-cells that frequently encounter their specific (self-) antigen will most likely undergo AICD. Whereas it is not easy to quantify this mechanism in a healthy individual, we may estimate the importance of this regulatory event by the analysis of experimental autoimmune disease models and autoimmune disease patients. Thus, it has been found that mice lacking functional CD95 (lpr) and CD95L ( gld) develop a severe lupus-like autoimmune disease, with lymphoadenopathy and high numbers of autoreactive $\mathrm{T}$ and $\mathrm{B}$ cells $[33,39]$. The fact that lack of CD95/CD95L-mediated suicide is responsible for this spontaneous development of autoimmune disease is demonstrated by the observation that transgenic expression of functional CD95 can reverse autoimmunity in $l p r$ mice $[40,41]$. Similarly, different studies have shown that many patients with autoimmune diseases have genetic defects in either the CD95, or the CD95L gene. Heterozygous mutations result in a mild form; homozygous mutations in the CD95 or CD95L locus lead to a more severe form of the disease [42-44]. 


\section{Regulation of AICD, CD95- and TNF receptor-induced apoptosis}

It appears obvious that CD95- and TNF receptorinduced apoptosis has to be a rather tightly regulated event. In particular, TNF receptor I and II are ubiquitously expressed. Therefore, low levels of inflammation would cause significant tissue damage due to systemically released TNF- $\alpha$. Similarly, in the absence of negative regulatory components in the CD95 signalling cascade, activated B- and T-lymphocytes would be immediately eliminated by CD95-induced apoptosis, and our body would become defenceless because of immune incompetence. Therefore, efficient immune homeostasis requires a fine-tuned balance between inhibition of apoptosis and development of immune effector functions on one hand, and induction of apoptosis, inhibition of tissue damage and development of autoimmune diseases on the other. What is the nature of these negative regulatory elements that allow this fine-tuning of immune responses? Could their inappropriate expression represent initiation for the development of autoimmune disease? Increasing evidence indicates that similar mechanisms regulate normal T-cell homeostasis and autoimmune T-cells, with the slight, but significant difference that inhibitors of apoptosis appear not to be appropriately down-modulated during the onset of autoimmune disorders, thus leading to an extended expansion of autoreactive T-cells. Therefore, the modulation of expression and/or activity of these inhibitors offers an attractive potential target for the treatment of T-cellmediated autoimmune diseases.

Many inhibitors of apoptosis, and more specifically of TNF receptor - and CD95-induced apoptosis, have been identified. Whereas the protective effect of Bcl-2 on CD95-induced apoptosis and AICD is still controversial [45-54], both Bcl-2 and Bcl- $\mathrm{x}_{\mathrm{L}}$ certainly have an important function in maintaining the survival of developing $\mathrm{T}$ and B-lymphocytes and in the prevention of apoptosis induced by other agents in many different cell types. Their role in development of autoimmunity has not been fully explored, but circumstantial evidence suggests, at best, a limited role in these processes, since transgenic overexpression of $\mathrm{Bcl}-2$ and $\mathrm{Bcl}-\mathrm{x}_{\mathrm{L}}$ protects $\mathrm{T}$-cells from dexamethasone and radiation-induced apoptosis, whereas AICD in vitro and in vivo remains normal $[50,55]$. However, development of autoimmunity in $l p r$ mice appears to be potentiated by transgenic overexpression of $\mathrm{Bcl}-2[56,57]$. This indicates that enhanced survival of immature T-cells by Bcl-2 overexpression may result in elevated numbers of mature potentially autoreactive T-cells, unless they are eliminated by AICD.

Hence, inhibitory molecules preventing induction of apoptotic pathways operative in T-cells, such as CD95 and TNF-receptor-induced apoptosis, may thus represent prime candidates to cause inappropriate survival and expansion of autoreactive T-cells in the periphery. Of particular interest are the recently identified FAP-1 (Fas-associated protein-1) and FLIP (Flice-like inhibitory protein). FAP-1 has been identified by its association with the intracellular domain of CD95 [58]. Interestingly, this domain, consisting of the last 15 amino acids at the N-terminal end, has been found to exhibit a regulatory function [59]. Whereas CD95 ligation does not always trigger apoptosis in certain cell types, due to specific inhibition of CD95 signalling pathways, truncation of this regulatory domain leads to accelerated apoptosis upon CD95 crosslinking. Therefore, this domain may interact with potential inhibitors of the CD95 signalling cascade. Indeed, Sato and colleagues have shown that overexpression of FAP-1 in Jurkat cells reduces apoptosis upon CD95 ligation [58]. Up to now, it is unclear whether FAP-1 inhibits via sterical hindrance of CD95 trimerisation and assembly of the signalling complex (FAP-1 has a molecular weight of approx. $200 \mathrm{kd}$ ), or through its tyrosine phosphatase activity [58].

One of the first events in the CD95 signalling cascade is the recruitment of FADD (Fas-associated death domain) and caspase 8 (a cysteine protease) to the receptor and the proteolytic processing of caspase 8 to its mature form [60]. Cellular FLIPs are molecules with strong sequence homology to caspase 8 , which appear to compete for FADD binding and therefore specifically block CD95 and TNF receptor I signalling and apoptosis at a very early stage [61,62]. Thus, FLIP would fulfil the above described requirements for a specific regulator of normal immune homeostasis and autoimmunity. Indeed, it has been found that IL-2, which promotes sensitivity to AICD in T-cells, also down-modulates FLIP and FAP-1 expression [63,64]. Alternatively, other molecules that may affect FLIP activity (e.g., by regulating its expression or recruitment to the receptor) could be responsible for the reduced sensitivity to AICD in T-cells [65]. Regardless of the identity and relative importance of these inhibitors, they appear to have one thing in common, which may allow to target efficiently these molecules. The 
Figure 2: Signalling events in CD95- and TNF receptor I-mediated apoptosis: Trimerisation of CD95 recruits the adaptor molecule FADD and subsequently the immature form of caspase 8 (pro-caspase 8 ). This recruitment to the receptor leads to proteolytic activation of caspase 8 and down-stream caspases, initiating the apoptosis machinery. Similarly, binding of TNF- $\alpha$ to TNF receptor I induces recruitment of the adaptor molecules TRADD and FADD, and activates caspase 8 . CD95 signalling is prevented by FAP-1, by a so-far unknown mechanism, whereas FLIP competes for pro-caspase 8 recruitment to the receptor complex. DD: Death domain; DED: Death effector domain.

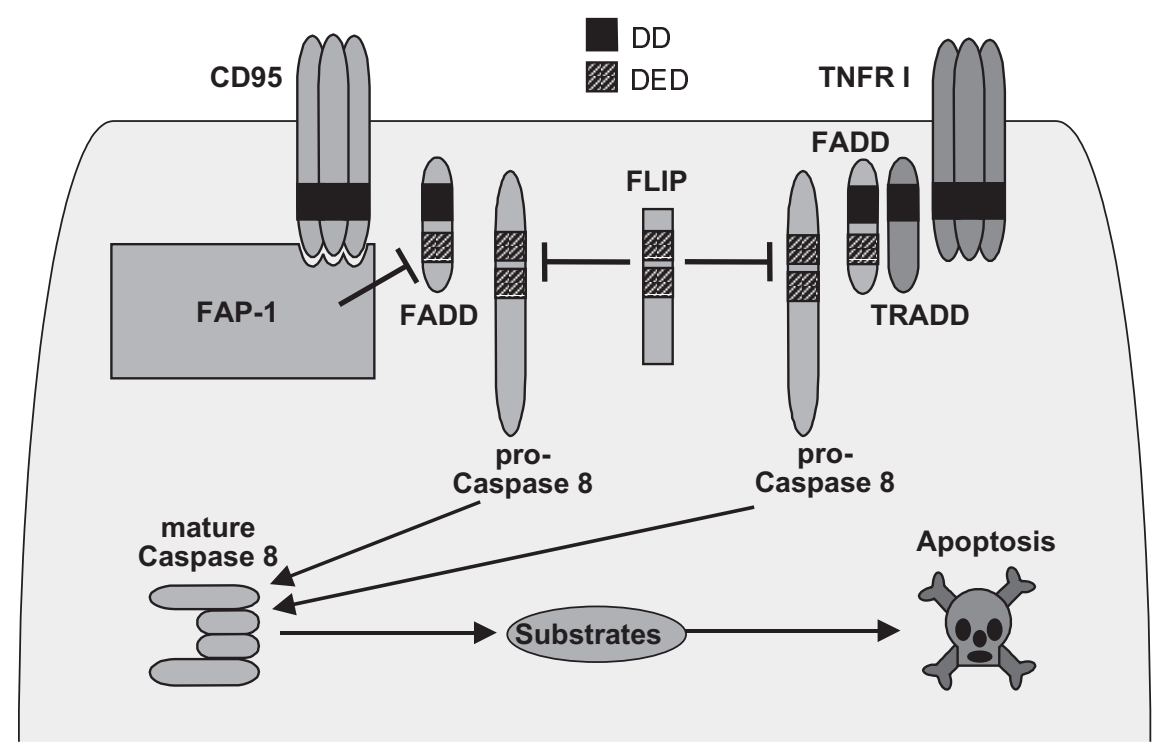

Figure 3: Chemical structure of bisindolylmaleimide VIII (Bis VIII).<smiles></smiles>

relevant inhibitors of CD95- and TNF receptorinduced apoptosis must have a rather limited half-life, since cells which are resistant to TNF- $\alpha$ or CD95 ligation (e.g. L929 cells and day 2 T-cells blasts, respectively) become sensitive to apoptosis induction after treatment with low concentrations of RNA and protein synthesis inhibitors. Since this effect is rather well distributed among different cell types, it is suggested that very few inhibitory molecules, ubiquitously expressed, may be responsible for the resistance of resting (or autoimmune) T-cells to TNF receptor- or CD95- induced cell death. Thus, this 'central blocker' of death receptor-induced apoptosis offers a prime target for drug development (see Figure 2).

\section{Bisindolylmaleimide VIII: a novel and prototypic drug for the treatment of autoimmune diseases}

A significant step towards specific inhibition of such a 'central blocker' was achieved in a recent study by Zhou and co-workers [66]. They have noticed that the protein kinase $\mathrm{C}$ (PKC) inhibitor, bisindolylmaleimide VIII (Bis VIII) (see Figure 3) sensitises different tumour lines, as well as primary T-cell lines for CD95and TNF receptor-induced apoptosis. This observation had been made previously by other research groups [67]. However, it was the accomplishment of Zhou and colleagues that demonstrated that inhibition of this 'central blocker' of death receptor-induced apoptosis can inhibit development of autoimmune diseases in vivo. Thus, in two experimental models of autoimmune disease, EAE and adjuvant-induced arthritis, disease progression and severity were strongly reduced by the repeated administration of Bis VIII. The attenuation of autoimmune disease development is most likely mediated by enhancing the T-cell's own suicide program (AICD), since death, and therefore inhibition of the immune response, are restricted to antigen-specific T-cells [66]. 
How does Bis VIII work? Will the understanding of its mode of action help us to identify the relevant targets for the treatment of autoimmune diseases? Whereas Bis VIII has been described so far as a potent inhibitor of PKC activation, the study by Zhou et al. indicates that the death-receptor sensitising activity of Bis VIII may not necessarily be related to PKC inactivation. Among different PKC inhibitors, only few were capable of sensitising target cells for CD95-induced apoptosis. Interestingly, however, all of those that exhibit a CD95 sensitising activity were bisindolylmaleimide derivatives [66]. Different explanations may account for this selective acceleration of CD95-induced apoptosis by bisindolylmaleimides: either bisindolylmaleimides have additional, yet unidentified, targets (unrelated to PKC) responsible for inhibition of death receptor induced apoptosis; alternatively, these drugs act more efficiently on distinct members of the large number of different PKCs. PKCs are a family of serine/threonine protein kinases, with different requirements for $\mathrm{Ca}^{2+}$ and activation by diacylglycerol. Certain isoforms have been implicated in distinct signalling pathways. Similarly, they appear to be differentially expressed in different tissues and cell types [68]. Thus, it is tempting to speculate that inhibitory effects on death receptorinduced apoptosis are mediated by distinct PKC isoforms which are efficiently antagonised by Bis VIII or related drugs. In favour of this hypothesis is the observation that phorbolmyristate acetate (PMA), a synthetic analogue of diacylglycerol, activates different PKCs and also attenuates anti-CD95-induced apoptosis in lymphoid tumours (see [69] and own unpublished results). However, PMA also inhibits UV-induced apoptosis in this cell type ( $\mathrm{T}$ Brunner, unpublished results). By contrast, Bis VIII activity appears to be restricted to death receptor-induced apoptosis, and does not affect dexamethasone- and $\gamma$-radiation-mediated death [66]. Therefore, the anti-apoptotic effect of PMA and the pro-apoptotic effect of Bis VIII could be unrelated. Alternatively, PMA could affect the CD95/CD95L-mediated signalling cascade of UV-induced apoptosis [70,71]. Thus, UV radiation induces expression of CD95L and subsequent apoptosis.

To date, no satisfying explanations for the activity of Bis VIII on AICD has been found. However, the hypothesis that distinct isoforms of PKC may be blocked by Bis VIII is attractive for several reasons. PKC has been shown to activate the transcription factor NFKB [68]. Similarly, many different stimuli, including TCR ligation and TNF- $\alpha$, can activate PKC and induce resistance to subsequent CD95-and TNF receptor-induced apoptosis, respectively [72-75]. In particular, the activation of NFKB appears to be an important step for the activation of genes intimately involved in mediating cell survival [74-77]. One family of NFkB-regulated target genes are the so-called inhibitors of apoptosis (IAPs), i.e., cellularly or virally encoded proteins which efficiently block apoptosis in response to different stimuli, as well as to TNF- $\alpha$ and CD95L [78-81]. Most signalling pathways inducing apoptosis involve the activation of a family of cysteine proteases (caspases) that either cleave additional immature forms of other family members, thus activating a cascade, or cleave proteins involved in cell structure or DNA replication [82]. Interestingly, IAPs can block apoptosis at the level of caspase activation through direct interaction with these caspases. Thus, c-IAP-1 and c-IAP-2 can bind and inactivate the two effector caspases, 3 and 7 [83]. By blocking NFKB activation, Bis VIII may regulate apoptosis through inhibition of IAP gene transcription, thus facilitating caspase activation. Recently, Wang and colleagues [77] have shown that NFKB induces expression and activation of TRAF1, TRAF2 (two signalling molecules of TNF receptor I and II) and C-IAP-1 and -2, and that these molecules are capable of blocking TNF- $\alpha$-induced apoptosis at the level of caspase 8 activation. Since caspase 8 is the most proximal caspase in the TNF receptor I and CD95 signalling cascade, it is suggested that these molecules directly act on the activation of this caspase, and that CD95-induced apoptosis could also be blocked by this mechanism (see Figure 4 ).

Several other mechanisms may account for the effect of Bis VIII on AICD and amelioration of autoimmunity in vivo. Whereas the identification of the signalling events that are affected by Bis VIII is mandatory for the development of more specific therapeutic approaches, the results obtained so far with this prototypical drug are highly encouraging for future development of similar drugs and strategies. Analogous to the process of tolerance induction using (auto-) antigenic peptides, the initial trigger leading to this functional unresponsiveness remains the antigen-specific activation of the T-cell. The specific activation of these sensitised T-cells leads to the expression of CD95 and CD95L and subsequent apoptosis. The auto-antigen involved in the disease does not have to be identified. Thus, the concept of enhancing the T-cells' own suicide program, which 
Figure 4: Possible mechanism of Bis VIII in enhancing AICD: T-cell receptor $\alpha \mathrm{TCR}$ ) activation and other signals activate protein kinase $\mathrm{C} \alpha \mathrm{PKC}$ ), and lead to the phosporylation of IKB and the activation of the transcription factor NFKB. NFkB may regulate expression of inhibitors of apoptosis, such as cIAP, FLIP or FAP-1. Bis VIII may interfere with resistance to apoptosis induction by specific inhibition of PKC and thus inhibition of NFKB activation.

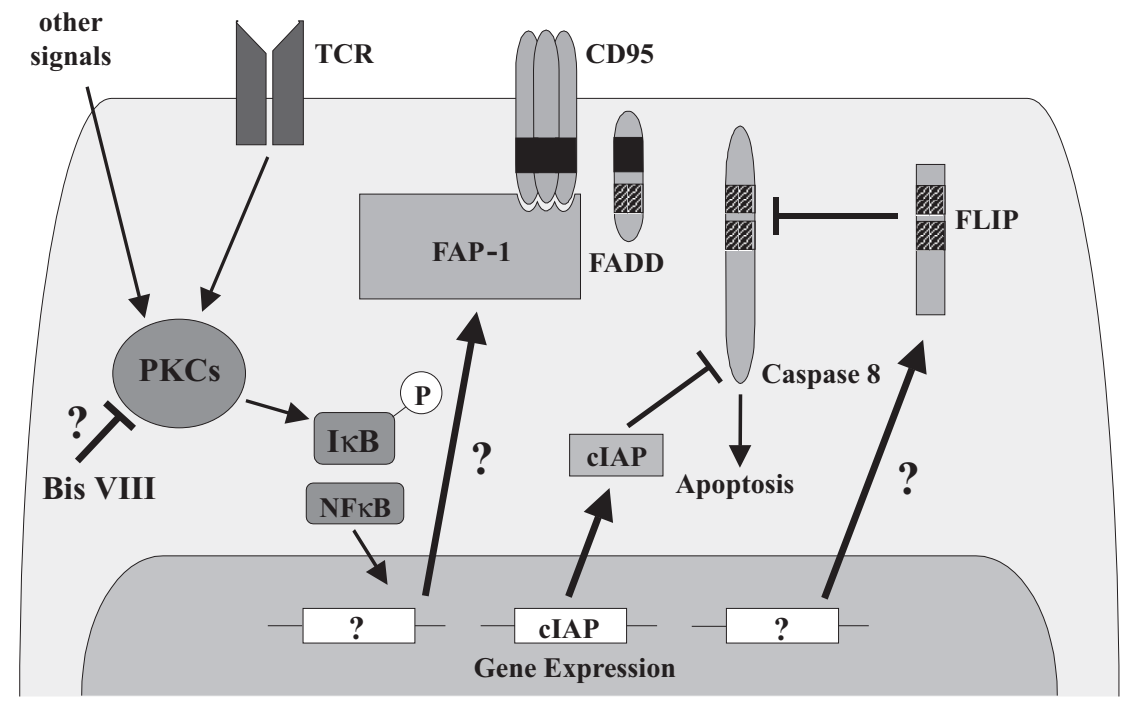

already exists but is impaired in autoimmune disease, may be an effective treatment of autoimmune diseases [84]. Zhou and colleagues [66] have demonstrated the beneficial effect of Bis VIII on two different experimental models of $\mathrm{CD}^{+}{ }^{+}$T-cell-mediated autoimmune diseases. Although it still remains to be formally demonstrated, Bis VIII may also ameliorate immunopathologies that involve cytoxic $\mathrm{CD}^{+} \mathrm{T}$-cells, since TNF- $\alpha$-induced apoptosis, an essential components of AICD in $\mathrm{CD}^{+}$T-cells $[35,36]$, is also enhanced. In addition, other diseases that are also mediated by an overly aggressive T-cell-mediated immune response (e.g. graft-versus-host disease, allograft rejection, inflammatory bowel disease), might be effectively targeted by Bis VIII.

Therapies aiming at enhancing the death receptormediated suicide of autoreactive lymphocytes may not necessarily depend on the development of apoptosis-sensitising drugs, such as Bis VIII. We and others have noticed that polarised T-cells show differential sensitivity to AICD $[85,86]$. CD $4^{+}$T-lymphocytes may not only differentiate into functional subsets producing distinct cytokines, but may also develop differential sensitivity towards CD95 ligation. Thus, T-cells differentiated into the T-helper (TH) 1 phenotype produce interferon- $\boldsymbol{\gamma}$ (IFN- $\gamma$ ) and interleukin-2 (IL-2) and show enhanced sensitivity towards AICD and CD95, whereas TH2 cells express IL-4, IL-5, IL-10 and show relative resistance to AICD $[85,86]$. The enhanced resistance of TH2 cells over
TH1 is most likely mediated by the differentiationinduced expression of FAP-1, which inhibits CD95 signalling [86]. Similarly, other cytokines, such as IFN- $\boldsymbol{\gamma}$ and IL-2, have been shown to enhance the sensitivity of T-cells for AICD and CD95-mediated apoptosis. Most likely, IL-2 does so by down-modulating FAP-1 and FLIP expression [63,64]. Therapies designed to modulate the immune response by specifically blocking certain polarising cytokines may therefore provide results similar to those achieved by Bis VIII. However, such strategies, aiming at the differentiation of $\mathrm{CD}^{+}{ }^{+} \mathrm{T}$-cells towards AICD-sensitive TH1 cells, will also have to deal with problematic side effect of enhanced cytotoxicity and therefore tissue destruction mediated by TH1 cells.

Whereas the identification of the molecular targets of Bis VIII will allow the design of more specific drugs to be used in the treatment of autoimmune diseases, specific inhibition of known anti-apoptotic molecules has been already successfully applied in experimental in vitro and in vivo systems. Yanagisawa and colleagues have identified the amino acid sequence in the cytoplasmic tail of CD95 responsible for the interaction with FAP-1 [87]. Administration of a synthetic tripeptide, corresponding to this sequence, to FAP-1 expressing colon carcinoma cell lines, rendered them sensitive to CD95-induced apoptosis. Similarly, down-modulation of the anti-apoptotic 14:18 chromosome breakpoint gene product Bcr-Abl by antisense oligonucleotides, efficiently sensitised 
Figure 5: Potential pitfalls of Bis VIII-based immunotherapy: A) Bis VIII may not only facilitate the death of autoreactive T-cells, but may also lead to premature activation-induced cell death of pathogen-specific T-cells and thus cause immune incompetence and persistence of infection (APC = antigen-presenting cell). B) Bis VIII may sensitise tissue cells, such as intestinal epithelial cells for CD95-mediated apoptosis. Activation of intestinal lymphocytes would cause premature loss of epithelial cells and tissue damage. C) Bis VIII enhances activation-induced cell death of 'normal' autoreactive T-cells, but is ineffective in sensitising T-cells with genetic defects in the CD95 signalling cascade. D) Bis VIII sensitised tumour-infiltrating T-cells may be killed by tumour cell-expressed CD95L. Tumour cells may be protected from CD95-mediated suicide by down-modulation of the receptor. IEL: Intraepithelial lymphocyte.

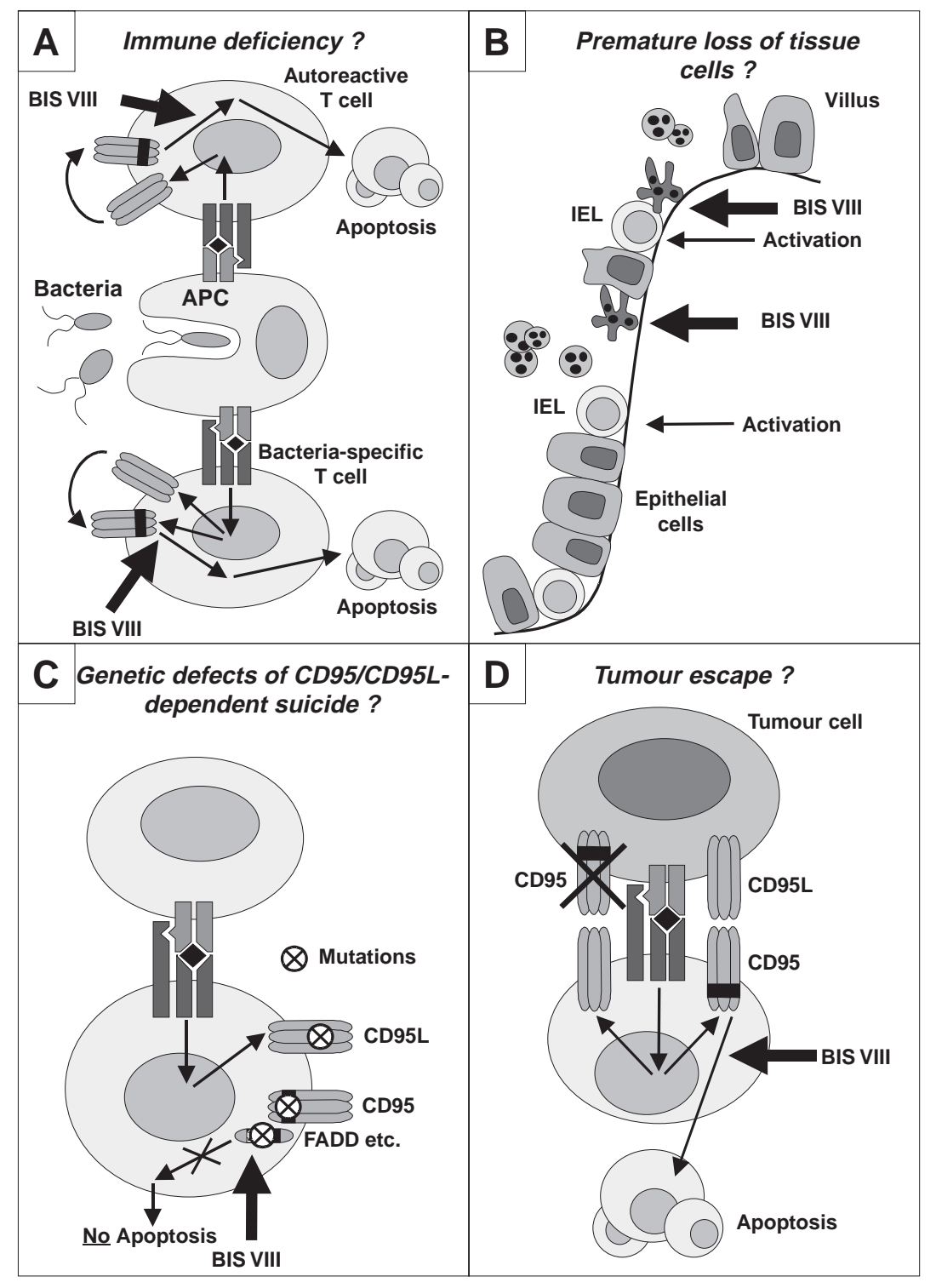

Otherwise resistant tumour cells to CD95 ligation [88]. In vivo targeting of $\mathrm{Bcl}-2$ with antisense oligonucleotides has been already successfully used to support chemotherapy of human malignant tumours transferred to SCID mice [89].

We must, however, be cautious: since Bis VIII sensitises all cells, not only autoreactive T-cells, the humoral and cellular immune responses against invasion could also be impaired. Thus, particular caution must be taken during such treatment with Bis VIII-like drugs to avoid uncontrolled infections with opportunistic germs and viruses. Similarly, Bis VIII will probably also sensitise tissue cells for TNF- $\alpha$ and CD95L-induced apoptosis. Thus, premature loss of intestinal epithelial cells or hepatocytes due to 
insufficient removal of activated T-cells may result in severe side effects [90,91]. An important caveat to the universal use of Bis VIII and similarly acting substances is the recent finding that many non-lymphoid tumours can express CD95L on their cell surface (reviewed in [92]) Although it is not completely clear as to the extent CD95L represents a tumour escape mechanism, Bis VIII may sensitise tumour-infiltrating T-cells to CD95L-induced apoptosis, and thus prevent an efficient antitumour immune response. In addition, the tumour would most likely survive this scenario, since many tumour cells have down-modulated CD95 on their cell surface, and are thus insensitive.

Other limitations for the use of Bis VIII-like drugs may be given by the molecular defects inherent to some of the autoimmune diseases to be treated. Bis VIII is effective in enhancing the cell's own suicide program. However, if certain components of the signalling cascade are irreversibly damaged, e.g. through genetic defects, Bis VIII may be completely ineffective in blocking autoimmunity. For example, many patients with ALPS (autoimmune lymphoproliferative syndrome) carry mutations in the CD95, CD95L or FADD gene $[42,93]$; apoptosis signalling is thus impaired, regardless of the sensitivity state of the cells. Similarly, patients suffering from SLE (systemic lupus erythematosus) and rheumatoid arthritis have elevated serum levels of soluble CD95, produced by alternative splicing of the CD95 mRNA [27,28,91]. Soluble CD95 efficiently binds cell surface CD95L and neutralises its apoptosis-inducing activity $[16,17]$. Thus, Bis VIII or drugs with a similar mode of action may be suitable for the treatment of certain autoimmune diseases, but may be ineffective or even dangerous for the treatment of others (see Figure 5).

\section{Conclusion and expert opinion}

Increasing evidence indicates that uncompromised death receptor-mediated suicide is crucial for the control of normal immune homeostasis and autoimmunity. The inappropriate survival of autoreactive T-cells due to resistance to this suicide program represents the most likely underlying cause of many autoimmune diseases. Therefore, molecules that regulate death receptor-mediated apoptosis are important targets for specific therapeutic approaches and the development of drugs aimed at reducing development of autoimmunity. From this perspective, Bis VIII represents a prototypic drug that shows very promising effects on the treatment of certain experimental autoimmune disease models and therefore strongly encourages further research on the specific activation of the cell's inherent suicide program. The beneficial effects of Bis VIII on autoimmune diseases may currently be overestimated, and potential pitfalls and side effects may become evident only at a later stage of research. However, the concept of enhancing the self-programmed demise of autoreactive T-cells may lead to more specific and more successful drugs and therapies for the treatment of autoimmunity.

\section{Acknowledgments}

The authors thank M Hodel for help with the preparation of the manuscript. This work is supported by grants from the Swiss National Science Foundation (31-54079.98) and Swiss Cancer League (SKL 617-2-98) to T Brunner.

\section{Bibliography}

Papers of special note have been highlighted as:

- of interest

- of considerable interest

1. DE MARIA R, TESTI R: Fas-FasL interactions: a common pathogenetic mechanism in organ- specific autoimmunity. Immunol. Today. (1998) 19(3):121-125.

2. MCDEVITT HO: The role of MHC class II molecules in susceptibility and resistance to autoimmunity. Curr. Opin. Immunol. (1998) 10(6):677-681.

3. O'GARRA A, STEINMAN L, GIJBELS K: CD4+ T-cell subsets in autoimmunity. Curr. Opin. Immunol. (1997) 9(6):872-883.

4. TIAN J, OLCOTT A, HANSSEN L et al: : Antigen-based immunotherapy for autoimmune disease: from animal models to humans? Immunol. Today. (1999) 20(4):190-195.

5. PARRY SL, HALL FC, OLSON J et al.: Autoreactivity versus autoaggression: a different perspective on human autoantigens. Curr. Opin. Immunol. (1998) 10(6):663-668.

6. KUCHROO VK, WEINER HL: Antigen-driven regulation of experimental autoimmune encephalomyelitis. Res. Immunol. (1998) 149(9):759-771; discussion 842-753, 855-760.

7. WALDMANN H, COBBOLD S: How do monoclonal antibodies induce tolerance? A role for infectious tolerance. Ann. Rev. Immunol. (1998) 16:619-644.

8. BRUNNER T, GENESTIER L: DATELINE Aix-les-bains. Cell Death Differ. (1998) 5(6):539-546.

9. SLOAN-LANCASTER J, ALLEN PM: Altered peptide ligand-induced partial $T$ cell activation: molecular 
mechanisms and role in $\mathbf{T}$ cell biology. Ann. Rev. Immunol. (1996) 14:1-27.

10. SINGER NG, MCCUNE WJ: Update on immunosuppressive therapy. Curr. Opin. Rbeumatol. (1998) 10(3):169-173

11. BUTZ EA, BEVAN MJ: Massive expansion of antigenspecific $\mathrm{CD8}+\mathbf{T}$ cells during an acute virus infection. Immunity. (1998) 8(2):167-175.

- Assessment of the extent of antigen-specific T-cell expansion

12. MURALI-KRISHNA K, ALTMAN JD, SURESH M et al.: Counting antigen-specific $\mathrm{CD8} T$ cells: a reevaluation of bystander activation during viral infection. Immunity (1998) 8(2):177-187.

- Assessment of the extent of antigen-specific T-cell expansion.

13. MOUNTZ JD, ZHOU $\mathrm{T}$, SU $\mathrm{X}$ et al.: The role of programmed cell death as an emerging new concept for the pathogenesis of autoimmune diseases. Clin. Immunol. Immunopathol. (1996) 80(3 Pt 2):S2-14.

14. VAN PARIJS L, ABBAS AK: Homeostasis and self-tolerance in the immune system: turning lymphocytes off. Science. (1998) 280(5361):243-248.

- Overview on the role of AICD in T-cell regulation.

15. O'REILly LA, STRASSER A: Apoptosis and autoimmune disease. Inflamm. Res. (1999) 48(1):5-21.

16. BRUNNER T, MOGIL RJ, LA FACE $\mathrm{D}$ et al:: Cell-autonomous Fas (CD95)/Fas-ligand interaction mediates activation-induced apoptosis in $\mathbf{T}$-cell hybridomas. Nature (1995) 373(6513):441-444.

- $\quad$ First demonstration of cell-autonomous suicide in T-cells.

17. BRUNNER T, YOO NJ, LA FACE D et al:: Activationinduced cell death in murine $T$ cell hybridomas. Differential regulation of Fas (CD95) versus Fas ligand expression by cyclosporin A and FK506. Int. Immunol. (1996) 8(7):1017-1026.

- $\quad$ First demonstration of cell-autonomous suicide in T-cells.

18. DHEIN J, WALCZAK H, BAUMLER C et al:: Autocrine T-cell suicide mediated by APO-1/Fas (CD95). Nature (1995) 373:438-441.

19. JU ST, PANKA DJ, CUI H et al.: Fas $\alpha$ CD95)/FasL interactions required for programmed cell death after $T$-cell activation. Nature (1995) 373:444-448.

20. ALDERSON MR, TOUGH TW, DAVIS-SMITH T et al: Fas ligand mediates activation-induced cell death in human T lymphocytes. J. Exp. Med. (1995) 181):71-77.

21. SU X, CHENG J, LIU W et al:: Autocrine and paracrine apoptosis are mediated by differential regulation of Fas ligand activity in two distinct Jurkat $T$ cell populations. J. Immunol. (1998) 160(11):5288-5293.

22. BRUNNER T, YOO NJ, GRIFFITH TS et al: : Regulation of CD95 ligand expression: a key element in immune regulation? Bebring Inst. Mitt. (1996) 97:161-174.
23. MARIANI SM, MATIBA B, BÄUMLER C et al:: Regulation of cell surface APO-1/Fas $\alpha$ CD95) ligand expression by metalloproteases. Eur. J. Immunol. (1995) 25:2303-2307.

24. SUDA T, HASHIMOTO H, TANAKA M et al:: Membrane Fas ligand kills human peripheral blood T lymphocytes, and soluble Fas ligand blocks the killing. J. Exp. Med. (1997) 186(12):2045-2050.

- Demonstration that membrane-bound and soluble CD95L have distinct functions.

25. TANAKA M, SUDA T, TAKAHASHI T et al:: Expression of the functional soluble form of human fas ligand in activated lymphocytes. EMBOJ. (1995) 14(6):1129-1135.

26. TANAKA M, ITAI T, ADACHI M et al:: Downregulation of Fas ligand by shedding. Nat. Med. (1998) 4(1):31-36.

27. Cheng J, ZHOU T, LIU C et al.: Protection from fas-mediated apoptosis by a soluble form of the fas molecule. Science (1994) 263:1759-1762.

- First demonstration that soluble CD95 is involved in autoimmune disease.

28. CASCINO I, FIUCCI G, PAPOFF G et al: : Three functional soluble forms of the human apoptosis-inducing fas molecule are produced by alternative splicing. $J$. Immunol. (1995) 154:2706-2713.

29. CASCINO I, PAPOFF G, DE MARIA R et al:: Fas/Apo-1 (CD95) receptor lacking the intracytoplasmic signaling domain protects tumor cells from fas-mediated apoptosis. J. Immunol. (1996) 156:13-17.

30. NAGATA S, SUDA T: Fas and fas ligand: lpr and gld mutations. Immunol.Today. (1995) 16(1):39-43.

31. RUSSELL JH, RUSH B, WEAVER C et al:: Mature T cells of autoimmune lpr/1pr mice have a defect in antigenstimulated suicide. Proc. Natl. Acad. Sci. USA (1993) 90(10):4409-4413.

- First demonstration of the effect of the lpr mutation in AICD.

32. MOGIL RJ, RADVANYI L, GONZALEZ-QUINTIAL R et al. Fas (CD95) participates in peripheral $T$ cell deletion and associated apoptosis in vivo. Int. Immunol. (1995) 7(9):1451-1458.

33. SINGER GG, CARRERA AC, MARSHAK-ROTHSTEIN A et al:: Apoptosis, fas and systemic autoimmunity: the MRL-1pr/1pr model. Curr. Op. Immunol. (1994) 6:913-920.

34. SINGER GG, ABBAS AK: The fas antigen is involved in peripheral but not thymic deletion of $T$ lymphocytes in $\mathbf{T}$ cell receptor transgenic mice. Immunity (1994) 1(5):365-371.

- Demonstrates the importance of CD95 in antigen-induced peripheral deletion.

35. SPEISER DE, SEBZDA E, OHTEKI T et al:: Tumor necrosis factor receptor $\mathbf{p 5}$ mediates deletion of peripheral cytotoxic T lymphocytes in vivo. Eur. J. Immunol. (1996) 26(12):3055-3060.

36. ZHENG L, FISHER G, MILLER RE et al:: Induction of apoptosis in mature $T$ cells by tumour necrosis factor. Nature (1995) 377:348-351. 
37. ASHKENAZI A, DIXIT VM: Apoptosis control by death and decoy receptors. Curr. Opin. Cell. Biol. (1999) 11(2): $255-260$

- Review on death-inducing receptors

38. KING C, SARVETNICK N: Organ-specific autoimmunity. Curr. Opin. Immunol. (1997) 9(6):863-871.

39. MOUNTZ JD, EDWARDS CK: Murine models of autoimmunity: T-cell and B-cell defects. Curr. Opin. Rheumatol. (1992) 4(5):612-620

40. WU J, ZHOU T, ZHANG J et al:: Correction of accelerated autoimmune disease by early replacement of the mutated lpr gene with the normal fas apoptosis gene in the T cells of transgenic MRL-lpr/lpr mice. Proc. Natl. Acad. Sci. USA (1994) 91:2344-2348.

41. ZHOU T, ILL CKE, MOUNTZ JD: Prevention of age-related $T$ cell apoptosis defect in CD2-fastransgenic mice. J. Exp. Med. (1995) 182:129-137.

42. RIEUX-LAUCAT F, LE DEIST F, HIVROZ C et al:: Mutations in fas associated with human lymphoproliferative syndrome and autoimmunity. Science. (1995) 268:1347-1349.

- $\quad$ One of the first demonstrations that genetic defects of CD95 can cause autoimmune disease.

43. VAISHNAW AK, ORLINICK JR, CHU JL et al:: The molecular basis for apoptotic defects in patients with CD95. J. Clin. Invest. (1999) 103(3):355-363

44. NAGATA S: Human autoimmune lymphoproliferative syndrome, a defect in the apoptosis-inducing Fas receptor: a lesson from the mouse model. J. Hum. Genet. (1998) 43(1):2-8

45. VAN PARIJS L, BIUCKIANS A, ABBAS AK: Functional roles of Fas and Bcl-2-regulated apoptosis of $\mathrm{T}$ lymphocytes. J. Immunol. (1998) 160(5):2065-2071.

46. PETSCHNER F, ZIMMERMAN C, STRASSER A et al.: Constitutive expression of Bcl-xL or Bcl-2 prevents peptide antigen- induced $T$ cell deletion but does not influence T cell homeostasis after a viral infection. Eur. $J$. Immunol. (1998) 28(2):560-569.

47. STRASSER A, HARRIS AW, HUANG DC et al: Bcl-2 and Fas/APO-1 regulate distinct pathways to lymphocyte apoptosis. $E M B O J$. (1995) 14):6136-6147.

48. SCHROTER M, LOWIN B, BORNER C et al:: Regulation of Fas (Apo-1/CD95)- and perforin-mediated lytic pathways of primary cytotoxic $T$ lymphocytes by the protooncogene bcl-2. Eur. J. Immunol. (1995) 25(12):3509-3513

49. IWAI OK, MIYAWAKI $T$, TAKIZAWA $T$ et al: Differential expression of bcl-2 and susceptibility to anti-Fasmediated cell death in peripheral blood lymphocytes, monocytes, and neutrophils. Blood (1994) 84(4):1201-1208.

50. MEMON SA, MORENO BM, PETRAK D et al.: Bcl-2 blocks glucocorticoid- but not Fas- or activation-induced apoptosis in a T cell hybridoma. J. Immunol. (1995) 155:4644-4652.
51. CHIU VK, WALSH CM, LIU C-C et al: Bcl-2 blocks degranulation but not fas-based cell-mediated cytotoxicity. J.Immunol. (1995) 154:2023-2032.

52. ALAM MK, DAVISON S, SIDDIQUI N et al.: Ectopic expression of Bcl-2, but not Bcl-xL rescues Ramos B cells from Fas-mediated apoptosis. Eur. J. Immunol. (1997) 27(12):3485-3491.

53. RODRIGUEZ I, MATSUURA K, KHATIB K et al: A bcl-2 transgene expressed in hepatocytes protects mice from fulminant liver destruction but not from rapid death induced by anti-Fas antibody injection. J. Exp. Med. (1996) 183(3):1031-1036.

54. KAWAHARA A, KOBAYASHI T, NAGATA S: Inhibition of Fas-induced apoptosis by Bcl-2. Oncogene (1998) 17(20):2549-2554.

55. STRASSER A: Life and death during lymphocyte development and function: evidence for two distinct killing mechanisms. Curr. Opin. Immunol. (1995) 7:228-234

56. TAMURA A, KATSUMATA M, GREENE MI et al.: Inhibition of apoptosis and augmentation of lymphoproliferation in bcl-2 transgenic Fas/Fas ligand-defective mice. Cell Immunol. (1996) 168(2):220-228.

57. REAP EA, FELIX NJ, WOLTHUSEN PA et al.: bcl-2 transgenic Lpr mice show profound enhancement of lymphadenopathy. J. Immunol. (1995) 155(11):5455-5462.

58. SATO T, IRIE S, KITADA S et al:: FAP-1: a protein tyrosine phosphatase that associates with fas. Science (1995) 268:411-415

- Identification of the first CD95-specific inhibitory molecule.

59. ITOH N, NAGATA S: A novel protein domain required for apoptosis. Mutational analysis of human fas antigen. J. Biol. Chem. (1993) 268(15):10932-10937.

60. MEDEMA JP, SCAFFIDI C, KISCHKEL FC et al:: FLICE is activated by association with the $\mathrm{CD} 95$ death-inducing signaling complex (DISC). Embo J. (1997) 16(10):2794-2804

- $\quad$ Biochemical analysis of the CD95 signalling complex.

61. SCAFFIDI C, SCHMITZ I, KRAMMER PH et al:: The role of c-FLIP in modulation of CD95-induced apoptosis. $J$. Biol. Chem. (1999) 274(3):1541-1548.

62. IRMLER M, THOME M, HAHNE $\mathrm{M}$ et al: Inhibition of death receptor signals by cellular FLIP. Nature (1997) 388(6638): 190-195.

- One of the first reports of the caspase 8 antagonsing molecule.

63. ZHOU YW, KOMADA Y, INABA H et al.: Down-regulation of Fas-associated phosphatase-1 (FAP-1) in interleukin- 2-activated T cells. Cell Immunol. (1998) 186(2):103-110

64. REFAELI Y, VAN PARIJS L, LONDON CA et al:: Biochemical mechanisms of IL-2-regulated Fas-mediated $\mathbf{T}$ cell apoptosis. Immunity (1998) 8(5):615-623.

- $\quad$ Role of cytokines in modulating sensitivity to AICD 
65. HITOSHI Y, LORENS J, KITADA SI et al.: Toso, a cell surface, specific regulator of Fas-induced apoptosis in T cells. Immunity (1998) 8(4):461-471.

66. ZHOU T, SONG L, YANG P et al.: Bisindolylmaleimide VIII facilitates Fas-mediated apoptosis and inhibits $\mathbf{T}$ cell-mediated autoimmune diseases. Nature Med. (1999) 5:42-48.

- $\quad$ First demonstration of the therapeutical use of a drug facilitating AICD.

67. RUIZ-RUIZ MC, IZQUIERDO M, DE MURCIA G et al:: Activation of protein kinase $\mathrm{C}$ attenuates early signals in Fas-mediated apoptosis. Eur. J. Immunol. (1997) 27(6):1442-1450.

68. TOKER A: Signaling through protein kinase C. Front Biosci. (1998) 1(3):D1134-1147.

- Thorough review on the protein kinase $\mathrm{C}$ family.

69. DREW L, KUMAR R, BANDYOPADHYAY D et al: Inhibition of the protein kinase $C$ pathway promotes anti-CD95-induced apoptosis in Jurkat cells. Int. Immunol. (1998) 10(7):877-889.

70. FRIESEN C, HERR I, KRAMMER PH et al:: Involvement of the CD95 (APO-1/Fas) receptor/ligand system in drug-induced apoptosis in leukemia cells. Nature Med. (1996) 2(5):574-577.

71. KASIBHATLA S, BRUNNER T, GENESTIER L et al:: DNA damaging agents induce expression of Fas ligand and subsequent apoptosis in $\mathrm{T}$ lymphocytes via the activation of NF-kappa B and AP-1. Mol. Cell. (1998) 1(4):543-551.

- Demonstration that DNA damage can activate the CD95L promoter.

72. SUDA T, TANAKA M, MIWA K et al: : Apoptosis of mouse naive $T$ cells induced by recombinant soluble Fas ligand and activation-induced resistance to Fas ligand. J. Immunol. (1996) 157(9):3918-3924.

73. KLAS C, DEBATIN K-M, JONKER RR et al: : Activation interferes with the APO-1 pathway in mature human $T$ cells. Int. Immunol. (1993) 5(8):625-630.

74. BEG AA, BALTIMORE D: An essential role for NF-kappaB in preventing TNF-alpha-induced cell death. Science (1996) 274(5288):782-784.

-

75. VAN ANTWERP DJ, MARTIN SJ, KAFRI T et al:: Suppression of TNF-alpha-induced apoptosis by NF-kappaB. Science (1996) 274(5288):787-789.

-

76. WANG CY, MAYO MW, BALDWIN AS, JR.: TNF- and cancer therapy-induced apoptosis: potentiation by inhibition of NF-kappaB. Science (1996) 274(5288): 784-787.

77. WANG CY, MAYO MW, KORNELUK RG et al.: NF-kappaB antiapoptosis: induction of TRAF1 and TRAF2 and c-IAP1 and c- IAP2 to suppress caspase-8 activation. Science (1998) 281(5383):1680-1683.

- Identification of the anti-apoptotic mechanism involving NFкB and c-IAPs.
78. CHU ZL, MCKINSEY TA, LIU L et al:: Suppression of tumor necrosis factor-induced cell death by inhibitor of apoptosis c-IAP2 is under NF-kappaB control. Proc. Natl. Acad. Sci. USA (1997) 94(19):10057-10062.

79. LI F, AMBROSINI G, CHU EY et al: Control of apoptosis and mitotic spindle checkpoint by survivin. Nature (1998) 396(6711):580-584.

80. TAMM I, WANG Y, SAUSVILLE E et al:: IAP-family protein survivin inhibits caspase activity and apoptosis. Cancer Res. (1998) 58(23):5315-5320.

81. DUDLEY E, HORNUNG F, ZHENG L et al:: NF-kappaB regulates Fas/APO-1/CD95- and TCR- mediated apoptosis of T lymphocytes. Eur. J. Immunol. (1999) 29(3):878-886

82. MARTIN SJ, GREEN DR: Protease activation during apoptosis: death by a thousand cuts? Cell (1995) 82(3):349-352.

83. LACASSE EC, BAIRD S, KORNELUK RG et al:: The inhibitors of apoptosis (IAPs) and their emerging role in cancer. Oncogene (1998) 17(25):3247-3259.

84. BRUNNER T, MUELLER C: Is autoimmunity coming to a Fas $\alpha$ t) end? Nature Med. (1999) 5(1):19-20.

85. RAMSDELL F, SEAMAN MS, MILLER RE et al.: Differential ability of Th1 and Th2 $T$ cells to express Fas ligand and to undergo activation-induced cell death. Int. Immunol. (1994) 6(10):1545-1553.

86. ZHANG X, BRUNNER T, CARTER L et al:: Unequal death in T helper cell (Th)1 and Th2 effectors: Th1, but not Th2, effectors undergo rapid Fas/FasL-mediated apoptosis. J. Exp. Med. (1997) 185(10):1837-1849.

87. YANAGISAWA J, TAKAHASHI M, KANKI $\mathrm{H}$ et al:: The molecular interaction of Fas and FAP-1. A tripeptide blocker of human Fas interaction with FAP-1 promotes Fas-induced apoptosis. J. Biol. Chem. (1997) 272(13):8539-8545.

- Demonstration of the therapeutical use of inhibitors of protein-protein interaction.

88. MCGAHON AJ, NISHIOKA WK, MARTIN SJ et al:: Regulation of the Fas apoptotic cell death pathway by Abl. $J$. Biol. Chem. (1995) 270(38):22625-22631.

89. JANSEN B, SCHLAGBAUER-WADL H, BROWN BD et al:: bcl-2 antisense therapy chemosensitizes human melanoma in SCID mice. Nature Med. (1998) 4(2):232-234.

90. LIN T, BRUNNER T, TIETZ B et al.: Fas ligand- mediated killing by intestinal intraepithelial lymphocytes. Participation in intestinal graft-versus-host disease. $J$. Clin. Invest. (1998) 101(3):570-577.

91. KONDO T, SUDA T, FUKUYAMA H et al.: Essential roles of the Fas ligand in the development of hepatitis. Nature Med. (1997) 3(4):409-413.

92. WALKER PR, SAAS P, DIETRICH PY: Role of Fas ligand (CD95L) in immune escape: the tumor cell strikes back. J. Immunol. (1997) 158(10):4521-4524.

- Review on CD95L expressed in different types of tumours. 
93. MARTIN DA, ZHENG L, SIEGEL RM et al: Defective CD95/APO-1/Fas signal complex formation in the human autoimmune lymphoproliferative syndrome, Type Ia. Proc. Natl. Acad. Sci. USA (1999) 96(8):4552-4557.

94. VAN LOPIK T, BIJL M, HART M et al:: Patients with systemic lupus erythematosus with high plasma levels of sFas risk relapse. J. Rheumatol. (1999) 26(1):60-67.

Thomas Brunner ${ }^{\dagger}$, Diana Arnold, Christoph Wasem, Jean A Laissue \& Christoph Mueller

${ }^{\dagger}$ Author for correspondence

Division of Immunopathology, Institute of Pathology, University of Berne, Murtenstrasse 31, 3010 Berne, Switzerland

Tel.: +41 31632 49 71; Fax: +41 3138187 64;

Email:tbrunner@pathology.unibe.ch 